Eur Neurol 1996;36:278-283

\begin{tabular}{|l|l|l|}
\hline Y. & Yoshihiro & Sato $^{\text {a }}$ \\
\hline H. & Hiroshi & Maruoka $^{\text {a }}$ \\
\hline Y. & Yoshiaki & Honda $^{\text {a }}$ \\
\hline T. & Takeshi & Asoh $^{\text {a }}$ \\
\hline Y. & Yukiko & Fujimatsu $^{\text {a }}$ \\
\hline K. & Kotaro & Oizumi $^{\text {a }}$ \\
\hline
\end{tabular}

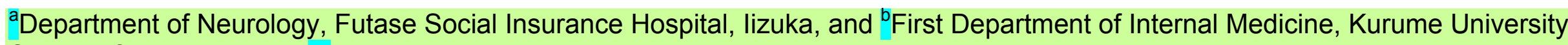
School of Medicine, Kurume, Japan

\title{
Development of Osteopenia in the Hemiplegic Finger in Patients with Stroke
}

\section{Key Words}

Stroke

Hemiplegia

Osteopenia

Hip fracture

Abstract

Previous studies have demonstrated that hip fractures in stroke patients occurred almost exclusively on the hemiplegic side. We examined the bone changes in the second metacarpal of the hemiplegic side in terms of microden-sitometric indices in 93 stroke patients with hemiplegia. All six indices indicated a significant decrease in bone mass on the hemiplegic side compared with the contralateral side. Differences in the indices between the hemiplegic and contralateral sides were correlated well with the duration of the illness and Brunstrom's stage for finger, arm, and leg. The same degree of osteopenia occurred in both small capsular and large hemispheric lesions. The same osteopenia was demonstrated in paralyzed and immobilized patients with myopathy. Thus a combination of weakness and immobilization is thought to be responsible for the osteopenia in the hemiplegic metacarpal bone. The osteopenia noted in the second metacarpal in the affected limb may account for the fact that hip fractures in stroke patients occur almost exclusively on the hemiplegic side. Dr. Yoshihiro Sato, Department of Neurology, Futase Social Insurance Hospital, 1243 Ikawa, lizuka 820 (Japan)

\section{Introduction}

It has been reported that $4-15 \%$ of patients with hip fractures have had previous cerebrovascular disorders and that $79-100 \%$ ofthe fractures occurred on the hemiplegic side [1-3]. This was explained by the development of disuse osteoporosis on the hemiplegic side that makes the bone more likely to break and the fact that hemiplegic patients with a stroke are prone to falls [4]. However, the pathogenesis of bone abnormalities on the hemiplegic side in patients with a previous stroke has not been fully elucidated $[5,6]$.

We examined microdensitometrically [7] the bone changes on the hemiplegic side in patients with a stroke and compared them to the contralateral side.

\section{Materials and Methods}

From 1991 to 1992,141 patients with hemiplegia due to stroke of more than 1 month's duration were admitted to our hospital. Of these, 48 were excluded because of an association with the shoulder hand syndrome $(\mathrm{n}=15)$, a previous history of stroke $(\mathrm{n}=2)$, or difficulty in taking finger radiographs due to impaired consciousness $(\mathrm{n}=31)$. Ofthe 93 patients studied, 57 were men and 36 were women, with ages ranging from 35 to 90 years (average: $69.8 \pm 7.2$, and $69.7 \pm 11.7$ years, respectively). The interval between the onset of stroke and the bone evaluation ranged from 32 to 8,156 days, with an average of 1,394 $\pm 1,863$ days. None ofthe patients had a history of hip fracture. A diagnosis of stroke was made on the basis of computed tomography (CT) in both the acute and chronic phases, as well as the clinical evaluation. In 8 patients whose duration of illness was greater than 5,475 days, however, a CT scan was performed only in the chronic phase, which revealed decreased attenuation. The decreased attenuation corresponded to the territory ofthe cerebral arteries in 3 of these patients. The strokes were classified as brain infarction $(n=67)$, brain hemorrhage $(n$ $=21)$, or unclassified $(n=5)$ according to

\section{KARGEH}

E-Mail karger@karger.ch Fax̣+416130612 34 http://www. karger.ch

(1) 1996 S. Karger|AG, Basel

Cross section of the metacarpal bone 


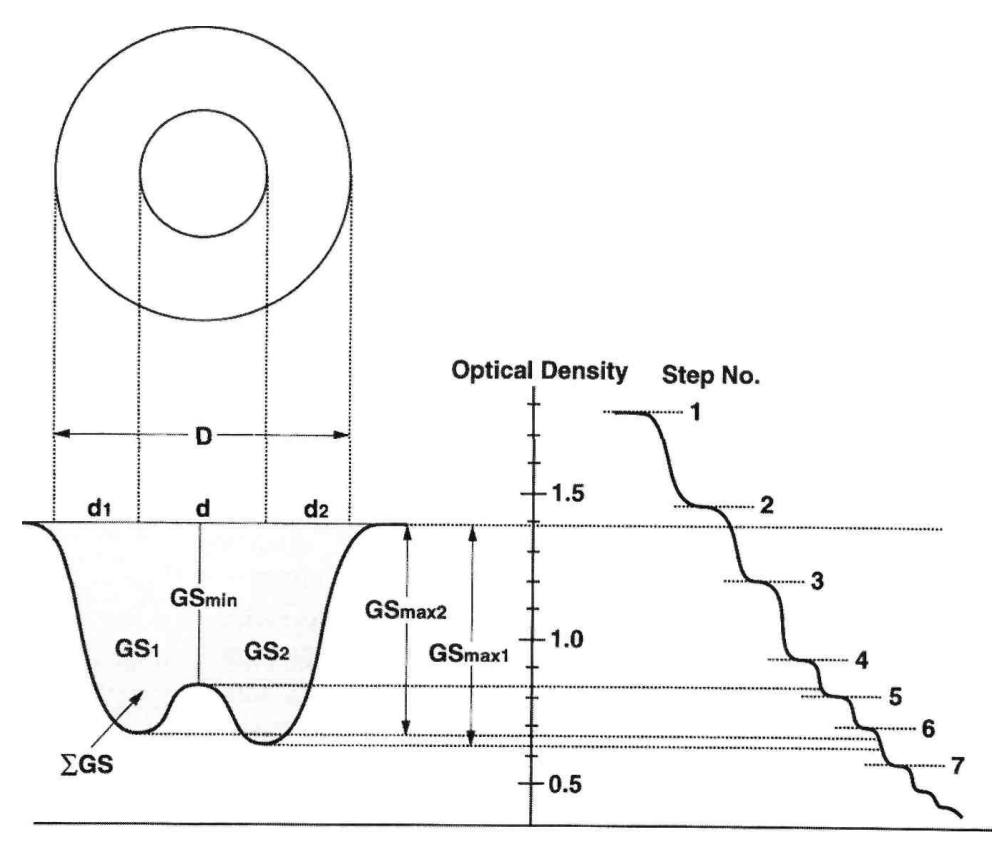

1) $\mathrm{MCI}=(\mathrm{d} 1+\mathrm{d} 2) / \mathrm{D}$

2) d (bone marrow width)

3) $\operatorname{GSmax}=(\operatorname{GSmaxl}+\mathrm{GSmax} 2) / 2$

4) GSmin

5) IGS/D

6) densitometer pattern index (1-9)

Fig. 1. Schematic of the MD method.

Ranking the bone cortex width $(\mathrm{d} 1+\mathrm{d} 2)$ and

the bone density, nine bone models (1-9)

were determined. Each index was ranked by

severity with a score of $0,1,2$, or 3 . From the sum of the scores of these six indices, the

MD score was calculated. $\mathrm{MCI}=$ Ratio of the

combined thickness of cortical bone ( $\mathrm{dl}$ and

d2) to D;

.

Optical density of metacarpal bone pattern

Aluminium step wedge

NINDS III. In the brain infarction group, a pure motor hemiparesis was due to a pontine lesion in 2 patients, and a capsular lacunar lesion in 21 patients. Cardioembolic stroke involving more than one cortical branch of the cerebral artery was present in 7 patients. A large hemispheric hemorrhage was present in 3 patients.

Clinical severity of the hemiplegia was evaluated using Brun-strom's staging classification [8]. Stage 1 is defined by no voluntary movement of the affected limb, while stage 6 is characterized by nearnormal strength in the arm, leg and each finger. In the fingers, an awkward weak hand grip is defined as stage 2, whereas the inability to release the hand grip is defined as stage 3 . Grasping of a small object between the thumb and the radial side of the index finger defines stage 4 , whereas spherical grasping ability is defined as stage 5 . In the arm, weak basic limb synergies are defined as stage 2 , whereas sufficient development of the synergies to show definite joint movement is defined as stage 3 . In stage 4 , an increasing number of movement combinations such as placing the dorsum of the hand in the lumbar region becomes possible. More difficult movement combinations such as raising the arm to a side-horizontal position with the elbow extended can be performed in stage 5. In the leg, 
slight voluntary movement, and mass hip-knee-ankle flexion are defined as stage 2 and 3, respectively. Voluntary flexion of the knee beyond $90^{\circ}$ is defined as stage 4 , whereas stage 5 is characterized by isolated knee flexion in the standing position with the hip fully extended. Overall, in the finger, hand, and leg, the average stages were $3.38 \pm 1.92,3.77 \pm 1.34$, and $3.45 \pm 1.75$, respectively.

37 patients suffered from right-sided and 56 from left-sided hemiplegia. Diabetes mellitus, which is known to result in osteopenia, was present in 19 patients. None of the patients had received drugs known to alter bone metabolism, including corticosteroids, thyroxin, or anticonvulsants.

For the determination of the bone changes on the hemiplegic side, plain radiographs of both hands were taken with an aluminum step scale, and were analyzed by a microdensitometer. The optical density at the center of the second metacarpal bone was read according to the method of loue et al. [7] (fig. 1). The bone width (D), bone marrow width (d), cortical width on the radial side (dl), cortical width on the ulnar side (d2), density at the center of the bone marrow (GSmin), peak density on the radial side (GSmaxl), and peak of density on the ulnar side (GSmax2) were measured. The cross section of the metacarpal bone was assumed to be a circle, and the cortical bone width ( $\mathrm{dl}$ and $\mathrm{d} 2$ ) was classified into three grades $(1,2 / 3$ and $1 / 3)$. The optical density of $\mathrm{dl}$ and $\mathrm{d} 2$, was also graded (1, $1 / 2$, and 0$)$. Nine bone models were prepared by drawing the metacarpal bone pattern according to the above parameters. These values were used to determine the following six indices: (1) the metacarpal index (MCI), (2) the bone marrow width index (d), (3) the center density index (GSmin), (4) the side density index, mean of the GSmaxl and GSmax2, (GSmax), (5) the index corresponding to the mineral content of the bone ( $2 \mathrm{GS} / \mathrm{D}, 2 \mathrm{GS}=$ the integrated value of the density area), and (6) the densitometer pattern index (1-9). There were 388 male and 315 female controls aged $30-90$ years (matched for age and sex). Changes in these indices in each sex and age group were defined as follows: changes of less than 1 standard deviation (SD) from the regression line of 703 healthy controls were expressed as a change of one point, less than $2 \mathrm{SD}$ as two points, and greater than $2 \mathrm{SD}$ as three points. From the sum of the scores of the six indices, the degree of bone loss was expressed as the microdensitometric (MD) score. Thus,

Osteopenia in Hemiplegic Stroke

Eur Neurol 1996;36:278-283

Table 1. Laterality of the bone changes in hemiplegic patients $(\mathrm{n}=93)$

Table 2. Comparison of the right and left hands of healthy controls $(\mathrm{n}=55)$

\begin{tabular}{|c|c|c|c|c|c|}
\hline & Hemiplegic side & Contralateral side & & Right & Left \\
\hline MCI & $0.386 \pm 0.094^{*}$ & $0.431 \pm 0.084$ & $\mathrm{MCI}$ & $0.477 \pm 0.089^{\mathrm{a}}$ & $0.469 \pm 0.079$ \\
\hline $\mathrm{d}$ & $5.524 \pm 1.033^{*}$ & $5.045 \pm 0.874$ & $\mathrm{~d}$ & $4.474 \mid \pm 0.842^{\bar{a}}$ & $4.439 \pm 0.791$ \\
\hline GSmax & $3.446 \pm 0.699^{*}$ & $3.790 \pm 0.550$ & GSmax & $3.930 \pm 0.441^{\bar{a}}$ & $3.781 \pm 0.397$ \\
\hline GSmin & $1.965 \pm 0.767^{*}$ & $2.399 \pm 0.643$ & GSmin & $2.599 \pm 0.627^{\bar{a}}$ & $2.639 \pm 0.623$ \\
\hline $\mathrm{XGS} / \mathrm{D}$ & $2.450 \pm 0.651^{*}$ & $2.801 \pm 0.506$ & $2 \mathrm{GS} / \mathrm{D}$ & $2.889 \pm 0.422^{\bar{a}}$ & $2.846 \pm 0.414$ \\
\hline Bone pattern & $3.925 \pm 2.789^{*}$ & $2.656 \pm 2.238$ & Bone pattern & $2.145 \pm 1.779^{\bar{a}}$ & $2.056 \pm 1.522$ \\
\hline MD score & $5.957 \pm 5.529^{*}$ & $2.630 \pm 3.340$ & MS score & $1.782 \pm 2.158^{\mathrm{a}}$ & $1.889+2.195$ \\
\hline
\end{tabular}

${ }^{*} \mathrm{p}<0.001$.

${ }^{\mathrm{a}}$ Not significant.

the lowered estimated values of MCI, GSmin, GSmax, and 2 GS/D, and conversely the elevated values of $\mathrm{d}$ and densitometer pattern index indicated the development and progression of osteopenia. The microdensitometric method permits the assessment of the degree of bone loss as indirect evidence of osteoporosis. The term osteopenia is used instead of osteoporosis in the text

To confirm the lack of laterality of abnormal values of the microdensitometric indices in healthy individuals, a total of 55 persons ( 25 males and 30 females) with an average age of $58.2 \pm 14.3$ years served as controls. To evaluate differences in bone abnormalities between stroke and paralyzed and immobilized patients without brain lesions, 6 patients with various types of myopathy (not on corticosteroids) were evaluated only by using the unilateral MD score ( 2 males and 4 females). They had a hand grip of less than $10 \mathrm{~kg}$, an average age of $35.2 \pm 11.3$ years, and a mean duration of illness of $7.5 \pm 6.4$ years.

In all of the patients, the six indices and the MD score were compared between the second metacarpals of the left and right sides. The six indices and MD score on the hemiplegic sides were compared between the 21 patients with pure motor hemiparesis due to lacunar infarction at the capsula interna and 10 patients with cardioembolic stroke or large hemispheric hemorrhage. The same comparison was made on the hemiplegic side between 19 diabetic and 74 nondiabetic patients.

The results were expressed as the mean \pm standard deviation. For statistical analysis, means were compared using the $t$ test for paired or unpaired samples. Fisher's exact test was used when appropriate. To examine between each index and the duration of illness and severity of the palsy, Spearman's rank correlation coefficients were calculated, p values of less than 0.05 were accepted as statistically significant.

\section{Results}

Table 3. Correlation between the hemi-plegic-nonhemiplegic differences and the duration of illness $(n=93)$

\begin{tabular}{lll}
\hline & \multicolumn{1}{c}{$\begin{array}{c}\text { Spearman's } \\
\text { rank correlation } \\
\text { coefficacy }\end{array}$} & p value \\
& 0.413 & 0.0001 \\
MCI & 0.352 & 0.0008 \\
d & 0.162 & 0.1211 \\
GSmax & 0.288 & 0.0058 \\
GSmin & 0.272 & 0.0092 \\
2 GS/D & 0.269 & 0.0218 \\
Bone pattern & 0.338 & 0.0014 \\
MD score & 0.38 \\
\hline
\end{tabular}


healthy controls (table 2). The MD score and the six indices on the intact side in the stroke patients were intermediate between those on the hemiplegic side and those in the controls (tables 1,2 ).

MD Score in Patients with Myopathy All 6 patients with myopathy had an MD score greater than 4, with a mean of $6.17 \pm 1.31$.

\section{Laterality of Abnormal Values of Bone Changes}

(tables 1,2)

All of the estimated values of the six indices and the MD score on the hemiplegic side differed significantly from those on the contralateral intact side (table 1 ), while the values were not different in the two hands of the

Relationship between the Hemiplegic-Nonhemiplegic Differences and the Duration of Illness (table 3) A strongly positive correlation was found between the leftright difference in the indices and MD score (except GSmax) and the duration of illness, suggesting continuous progression of osteopenia on the hemiplegic side.

280

Eur Neurol 1996;36:278-283

Sato/Maruoka/Honda/Asoh/Fujimatsu/ Oizumi

Table 4. Correlation between the hemiplegic-nonhemiplegic differences and Brunstrom's stage $(n=93)$

Table 5. Comparison of bone changes due to small lesions of the capsula interna with those due to large hemispheric lesions

\begin{tabular}{|c|c|c|c|}
\hline \multirow{3}{*}{$\overline{\mathrm{MCI}}$} & Finger & Upper limb & Lower li \\
\hline & -0.236 & -0.248 & -0.199 \\
\hline & $(0.013$ & $(0.0105)$ & $(0.0307)$ \\
\hline $\mathrm{d}$ & $\begin{array}{r}-0.281 \\
(0.0036\end{array}$ & $\begin{array}{l}-0.287 \\
\quad(0.0033)\end{array}$ & $\begin{array}{l}-0.196 \\
(0.0328)\end{array}$ \\
\hline GSmax & $\begin{array}{r}-0.464 \\
\quad(0.000\end{array}$ & $\begin{array}{l}-0.494 \\
\quad(0.0001)\end{array}$ & $\begin{array}{l}-0.260 \\
(0.0058)\end{array}$ \\
\hline GSmin & $\begin{array}{l}-0.424 \\
\quad(0.000\end{array}$ & $\begin{array}{l}-0.468 \\
\quad(0.0001)\end{array}$ & $\begin{array}{l}-0.260 \\
(0.0058)\end{array}$ \\
\hline SGS/D & $\begin{array}{r}-0.427 \\
\quad(0.000\end{array}$ & $\begin{array}{l}-0.464 \\
\quad(0.0001)\end{array}$ & $\begin{array}{l}-0.285 \\
(0.0027)\end{array}$ \\
\hline Bone pattern & $\begin{array}{r}-0.371 \\
\quad(0.000\end{array}$ & $\begin{array}{l}-0.424 \\
\quad(0.0001)\end{array}$ & $\begin{array}{l}-0.267 \\
(0.0009)\end{array}$ \\
\hline MD score & $\begin{array}{r}-0.482 \\
\quad(0.000\end{array}$ & $\begin{array}{l}-0.499 \\
\quad(0.0001)\end{array}$ & $\begin{array}{l}-0.293 \\
(0.0015)\end{array}$ \\
\hline $\begin{array}{l}\text { Small lesion } \\
(\mathrm{n}=21)\end{array}$ & & & $\begin{array}{l}\text { Large lesion } \\
(\mathrm{n}=10)\end{array}$ \\
\hline Age, years & & $68.6 \pm 8.5$ & $64.4 \pm 10.0^{\overline{\mathrm{a}}}$ \\
\hline Male/female & & $15 / 6$ & $6 / 4^{\bar{a}}$ \\
\hline $\begin{array}{l}\text { Duration of il } \\
\text { Brunstrom's s }\end{array}$ & $\begin{array}{l}\text { less, days } \\
\text { ale }\end{array}$ & $1,697+1,787$ & $1,458 \pm 2,418$ \\
\hline Finger & & $3.571 \pm 1.886$ & $2.200 \pm 1.874$ \\
\hline Arm & & $1.650 \pm 0.745$ & $2.000 \pm 0.943$ \\
\hline Leg & & $4.095 \pm 1.221$ & $2.800 \pm 1.619^{* *}$ \\
\hline MCI & & $0.376 \pm 0.103$ & $0.416 \pm 0.098^{2}$ \\
\hline d & & $5.694+1.186$ & $5.166 \pm 0.991^{2}$ \\
\hline GSmax & & $3.589 \pm 0.806$ & $3.346 \pm 0.643^{2}$ \\
\hline GSmin & & $2.076 \pm 0.859$ & $2.009 \pm 0.614$ \\
\hline 2GS/D & & $2.581+0.718$ & $2.437 \pm 0.524$ \\
\hline Bone pattern & & $4.095 \pm 3.192$ & $4.400 \pm 2.459^{2}$ \\
\hline MD score & & $6.476 \pm 5.980$ & $5.900 \pm 4.383$ \\
\hline
\end{tabular}

Values represent Spearman's rank correlation coefficacy (SRCC) with the $\mathrm{p}$ value in parentheses. SRCC was used to examine the relationship

between the left-right difference in the index and Brunstrom's stage. 
Relationship between the Hemiplegic-Nonhemiplegic Differences and Brunstrom 's Stage (table 4) A close correlation was demonstrated between the severity of palsy based on Brunstrom's stage and the left-right differences in the six indices and MD score calculated for the fingers, the upper and lower limbs.

Comparison of Bone Changes due to Pure Motor

Hemiparesis with Those due to a Large Hemispheric

Lesion (table 5)

There were no significant differences between the two patient groups with respect to age, sex, duration of illness, or Brunstrom's stage in the finger and arm. Musclar weakness of the leg (based on Brunstrom's stage) was significantly worse in the patients with large hemispheric lesions than in those with capsular lesions. There were no significant differences in any of the indices or the MD score between the two patient groups on the hemiplegic side.

Comparison of Bone Changes in Diabetic and

Nondiabetic Patients (table 6)

There were no significant differences with respect to age, sex, duration of illness, or Brunstrom's stage between the two patient groups. There were no significant differences in any of the indices or the MD score between the two patient groups in the hemiplegic side.

Table 6. Comparison of bone changes in diabetic and nondiabetic patients

$\operatorname{DM}(\mathrm{n}=19)$

Non-DM $(\mathrm{n}=74)$

\begin{tabular}{|c|c|c|}
\hline Male/female & $14 / 5$ & $42 / 32^{\mathrm{a}}$ \\
\hline Duration of illness, days & $1,116+1,591$ & $1,468 \pm 1.940 "$ \\
\hline Brunstrom's scale & & \\
\hline Finger & $4.111+1.367$ & $3.288+1.989^{\mathrm{a}}$ \\
\hline Arm & $3.778+1.629$ & $3.425 \pm 1.779^{\mathrm{a}}$ \\
\hline Leg & $4.000 \pm 1.188$ & $3.712 \pm 1.380^{\mathrm{a}}$ \\
\hline MCI & $0.399 \pm 0.075$ & $0.383 \pm 0.098^{\mathrm{a}}$ \\
\hline d & $5.406 \pm 0.959$ & $5.554 \pm 1.067^{\mathrm{a}}$ \\
\hline GSmax & $3.782 \pm 0.596$ & $3.383 \pm 0.703^{\mathrm{a}}$ \\
\hline GSmin & $2.327 \pm 0.714$ & $1.895 \pm 0.755^{\mathrm{a}}$ \\
\hline SGS/D & $2.739 \pm 0.562$ & $2.394 \pm 0.656^{\mathrm{a}}$ \\
\hline Bone pattern & $3.056 \pm 2.363$ & $4.055 \pm 2.847^{\overline{\mathrm{a}}}$ \\
\hline MS score & $4.389+4.828$ & $6164+5540^{\bar{a}}$ \\
\hline
\end{tabular}

DM $=$ Diabetes mellitus. Not significant.

\section{Discussion}

There are few reports of bone changes on the hemiplegic side in small numbers of patients with a stroke [5, 6], despite the fact that hip fractures almost exclusively occur in this location [1-3].

The present study, using the MD method of analysis of radiographs, confirmed a reduction of bone mass on the hemiplegic side when compared with the contralateral side. As the normal values of each index vary according to sex and age, we used the contralateral intact side as a control to detect abnormalities in the hemiplegic side. The MD method is based on a principle similar to that used in the photon absorption technique using X-rays instead of y-rays. The validity of this simple method has been reported by Inoue et al. [7]. They demonstrated, using iliac bone, a positive correlation between the five indices estimated by the MD method and the severity of osteoporosis as evaluated by photon absorptiometry and confirmed by pathologic examination.

It is noteworthy that a positive correlation was observed between the duration of illness and the differences between the hemiplegic and nonhemiplegic side. Moreover, a close correlation was found between the severity of the palsy, not only in the finger but also in the lower and upper limbs, and the differences between the hemiplegic and normal sides. It was 
also found that the same degree of osteopenia occurred in association with both small capsular and large hemispheric lesions. This indicates that size or site of the cerebral lesion is unrelated to the degree of osteopenia.

Several studies have demonstrated a loss of bone tissue during immobilization such as prolonged bed rest or spinal cord injury [9-11]. Biering-Sorensen et al. [11] demonstrated decreased bone mineral content of the lower extremities, and an unchanged bone mineral content in the distal forearm in patients with spinal cord injuries manifested as paraplegia. In the present study, a close correlation was found between the severity of the palsy and the degree of bone changes, which is in agreement with the findings in the patients with paraplegia. Decreased voluntary muscle power may account for a significant proportion of the osteopenia on the hemiplegic side since the existence of osteopenia was confirmed in patients with myopathy. It is concluded that the pathogenesis of the osteopenia on the hemiplegic side is a combination of muscular weakness and immobilization. Similar bone abnormalities may develop in the hemiplegic femoral neck as well as the finger, as evidenced by the positive correlation between Brunstrom's stage in the leg and the ob-

served differences in the indices in the finger. The reason why hip fractures occur almost exclusively in the femoral neck on the affected side may be explained by osteopenia.

The fact that the values of the contralateral side were intermediate between the hemiplegic side and those in the control subjects is not explained by differences in age and sex between the two groups because the MD score is absolute. In the hemiplegic state after a stroke, the dependence on professional care for the activities of daily living may result in a decrease in mobility of the intact limb. Therefore, mild contralateral osteopenia may exist parallel to the degree of immobilization of the intact limb.

Diabetes mellitus has been shown to be an important factor in the development of osteopenia [12] and is known to be a significant risk factor for stroke $[13,14]$. In the present study, however, there were no significant differences in any of the indices or the MD score between the two patient groups in the hemiplegic side. The reason is not known at present, and cannot be explained simply by differences in age, sex, or duration of the illness. A more detailed clinical investigation of a large number of patients is necessary to elucidate the relationship between diabetes mellitus and osteopenia on the hemiplegic side in stroke patients.

282

Eur Neurol 1996;36:278-283

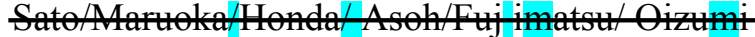

References

1 Mulley G, Espley AJ: Hip fracture after hemiplegia. Postgrad Med J 1979;55:264-265.

2 Poplingher AR, Pillar T: Hip fracture in stroke patients. Acta Orthop Scand 1985;56:226-227.

3 Chiu KY, Pun WK, Luk KDK, Chow SP: A prospective study on hip fractures in patients with previous cerebrovascular accidents. Injury 1992;23:297-299.

4 Peszczynski M: Prevention of falls in the hemi-plegic patients. Geriatrics 1956; 11:306-311.

5 Hodkinson HM, Brain AT: Unilateral osteoporosis in longstanding hemiplegia in the elderly. J Am Geriatr Soc 1967;15:59-64.

6 Goodman CR: Osteoporosis as early complication of hemiplegia. NY State J Med 1971;71: 1943-1945.

7 Inoue T, Kusida K, Miyamoto S, Sumi Y: Quantitative assessment of bone density on X-ray picture. J Jpn Orthop Assoc 1983;57:1923-1936.

8 Brunstrom S: Motor testing procedures in hemiplegia. Based on sequential recovery stages. Am J/PhysTher $1966 ; 46: 357-375$.

9 Donaldson CL, Hulley SB, Vogel JM, Hattner RS, Bayers JH, McMillian DE: Effect of prolonged bed rest on bone mineral. Metabolism 1970;19:1071-1084.

10 Chantraine A, Nusgens B, Lapiere CM: Bone remodeling during the development of osteoporosis in paraplegia. Calcif Tissue Int 1986; 38:323-327.

11 Biering-Serensen F, Bohr HH, Schaadt OP: Longitudinal study of bone mineral content in the lumbar spine, the forearm and the lower extremities after spinal cord injury. Eur J Clin Invest 1990;20:330-335.

12 Ishida H, Seino Y, Matsukura S, Ikeda M, Yawata M, Yamashita G, Ishizuka S, Imura H: Diabetic osteopenia and circulating levels of vitamin D metabolites in type 2 (non-insulin-dependent) diabetes. Metabolism 1985;34: 797-801.

13 Davis PH, Dambrosia J, Schoenberg BS, Schoenberg DG, Pritchard DA, Lilienfeld AM, Whisnant JP: Risk factors for ischemic stroke: A prospective study in Rochester, Minnesota. Ann Neurol 1987:22:319-327.

14 Boysen G, Nyboe J, Appleyard M, S0rensen PS, Boas J, Somnier F, Jensen G, Schnohr P: Stroke incidence and risk factors for stroke in Copenhagen, Denmark. Stroke 1988;19:1345-1353. 
Osteopenia in Hemiplegic Stroke

Eur Neurol 1996;36:278-283

283 